\title{
Non-Destructive Determination of In-Depth Profile of Brass
}

\author{
By K. Asami*
}

\begin{abstract}
A non-destructive in-depth analysis of a single phase brass has been done by a combination of X-ray induced AES, EPMA and X-ray diffraction. The analysing depth by this combination. covers the depth range from $1 \mathrm{~nm}$ to $10 \mu \mathrm{m}$. The anlytical composition $\bar{C}(L)$ by a method with analysing depth $L$ is converted to the true composition $C(L)$ at depth $L$ by using a certain composition-distribution model and the following relation;

$$
\bar{C}(L)=\int_{0}^{\infty} C(x) Y(x, L) \mathrm{d} x / \int_{0}^{\infty} Y(x, L) \mathrm{d} x
$$

where $Y(x, L)=\exp \left\{x \ln \left(1-G_{L}\right) / L\right\}$ for XAES and X-ray diffraction and $=\phi(\rho x, L) \cdot \exp (-\chi \rho x)$ for EPMA, $\phi(\rho x)$ is the ionization distribution function and $G_{L}$ is a constant. Samples were revealed to have a large dezincification zone at the depth less than $1 \mu \mathrm{m}$. The largest difference between $\bar{C}(L)$ and $C(L)$ in this case appeared in the depth range between $10^{-2}$ and $10^{-1} \mu \mathrm{m}$.
\end{abstract}

(Received December 11, 1979)

\section{Introduction}

One of the most familiar and handy techniques which will give an in-depth profile of an alloy with composition gradients ranging $10 \mu \mathrm{m}$ or more in depth is the electron probe micro-analysis (EPMA or XMA) scanning across the cross-section of the sample. This method is often adopted to the determination of the result of high temperature diffusion, the dezincification of brass and so on. This technique is, however, unable to apply to a case where an appreciable composition gradient is restricted to the surface region less than $1 \mu \mathrm{m}$ in depth. For such a case, a combination of Auger electron spectroscopy (AES) or X-ray photoelectron spectroscopy (XPS or ESCA) and ion sputter-etching has become an effective method. Secondary ion mass spectroscopy (SIMS) is also applicable. These methods are, however, always destructive and also accompanied by a problem of preferential sputtering of alloying components ${ }^{(1)}$. We are often encountered with cases where samples should not be destroyed and, therefore, it is preferable to develop a non-destructive analysing technique.

* The Research Institute for Iron, Steel and Other Metals, Tohoku University, Sendai 980, Japan.

Trans. JIM
Non-destructive analyses have been tried by AES and XPS with various take-off angles ${ }^{(2)(3)}$ utilizing the energy difference between $\mathrm{Al}$ and $\mathrm{Mg} K \alpha$ radiations $^{(4)}$, and the energy difference among core levels ${ }^{(5)}$. XPS methods are often useful for analysis of the very surface region, usually less than $10 \mathrm{~nm}$, but are impossible to probe into a deeper region because of the small escape depth of photoelectrons. Houska and his coworkers ${ }^{(6)} \sim(8)$ has developed an $\mathrm{X}$-ray diffraction technique for a non-destructive in-depth profile in the micron and submicron range by analysing the diffraction intensity bands. X-ray diffraction with various photon energies has also been used to detect the distribution of residual strain in steel ${ }^{(9)}$. This technique is applicable to the $\mu \mathrm{m}$ range depth analysis.

In this report, a non-destructive analytical method for determination of the in-depth composition profile over a range from $1 \mathrm{~nm}$ to $10 \mu \mathrm{m}$ is developed for $\alpha$-brass, combining $\mathrm{X}$-ray induced AES (XAES), EPMA and X-ray diffraction (XD).

\section{Experimental}

Two kinds of $\alpha$-brass specimens used for this work were the same as those used in a previous paper ${ }^{(10)}$. They were cut out from the final strip products; $0.25 \mathrm{~mm}$ thick, with the same

1980 Vol. 21 
bulk chemical composition, nominally $\mathrm{Cu}$ $35 \mathrm{wt} \% \mathrm{Zn}-0.03 \mathrm{wt} \% \mathrm{~Pb}-0.05 \mathrm{wt} \% \mathrm{Fe}$, but different in the surface dezinicification because of different production processes.

The $L_{3} M_{4,5} M_{4,5}$ Auger electrons of $\mathrm{Cu}$ and $\mathrm{Zn}$ were excited with $\mathrm{Mg} K \alpha$ radiation and their spectra were measured with an AEI ES200 electron spectrometer in a vacuum of $c a$. $4 \times 10^{-7} \mathrm{~Pa}$. Details concerning with the treatment of XAES data were the same as described in the previous paper ${ }^{(10)}$. EPMA was done with a JEOL U-3 type analyser with a take-off angle of $\pi / 6 \mathrm{rad}$ and a fixed absorption current of $2 \times 10^{-8} \mathrm{~A}$. The $K \alpha$ lines of $\mathrm{Cu}$ and $\mathrm{Zn}$ excited at acceleration voltage of 15,25 and $50 \mathrm{kV}$ were detected by a LiF crystal, and $L \alpha$ lines at 5 and $10 \mathrm{kV}$, by a RAP crystal. Contamination by carbon deposit during the measurement was lowered by mechanical scan of samples with a speed of $1 / 3 \mu \mathrm{m} / \mathrm{s}$. For the XD measurements, and $\mathrm{X}$-ray diffractometer was used with $\mathrm{Cu}$ $K \alpha_{1,2}$ and $\mathrm{Cr} K \alpha_{1,2}$ radiations. Composition was estimated from the lattice spacing measured, using the relationship between the composition and lattice constant reported by Beck and Smith ${ }^{(1)}$. Since the analysing depth of $\mathrm{XD}$ depends on the diffraction angle, the angles for (420) reflections by $\mathrm{Cu} K \alpha$ and (220) reflections by $\mathrm{Cr} K \alpha$ were adopted for the calculation of analysing depth.

\section{Analysing depths and corresponding composition}

The analysing depth, $L$, is defined in the same way as in the previous paper ${ }^{(10)}$, that is, "when the fraction $G_{L}$ of the total signal intensity is detectable from the thickness range $L$ of a homogenous substance of semi-infinite thickness, the thickness $L$ is the analysing depth". For convenience, $G_{L}$ is taken as $7 / 9$ because of the reason described later in this section. For XAES, the signal intensity of electrons, $\mathrm{d} I_{e}$, from a thin slab with thickness $\mathrm{d} x$ at depth $x$ (Fig. 1(a)) can be adequately approximated by the following equation because of the very small escape depth of Auger electrons compared with that of incident X-rays ${ }^{(12)}$;

$$
\mathrm{d} I_{e} \propto \exp (-x / \lambda \sin \theta) \cdot \mathrm{d} x,
$$
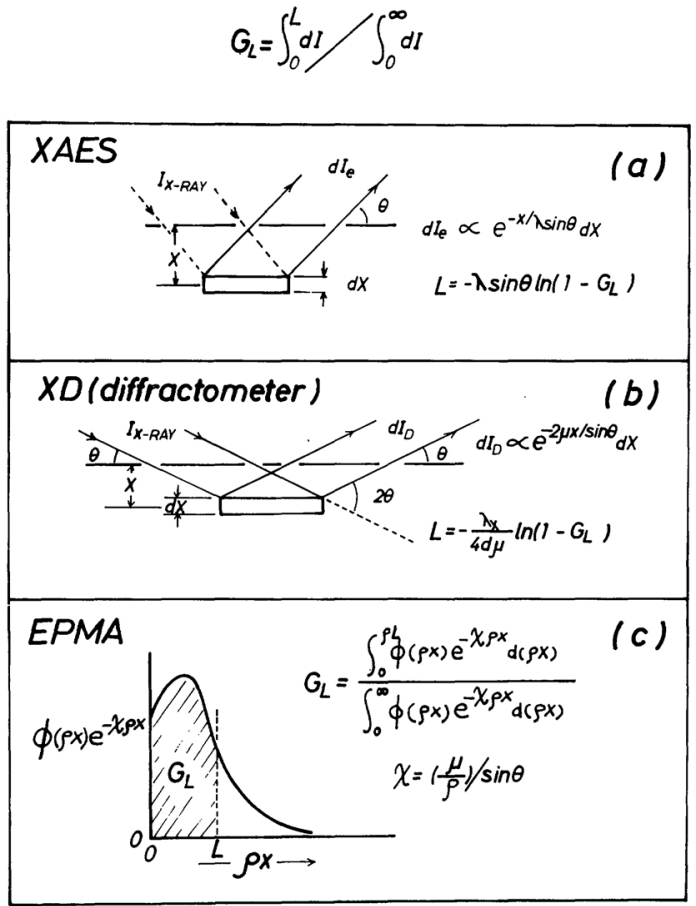

Fig. 1 Definition of analysing depth and its application to XAES (a), XD (b) and EPMA (c).

where $\lambda$ is the mean escape depth of the $\mathrm{Cu}$ and $\mathrm{Zn}$ Auger electrons and $\theta$, the take-off angle of Auger electrons. By the definition of the analysing depth $L$, it is easy to obtain the analysing depth for XAES, $L$ (XAES), as follows:

$$
L(\mathrm{XAES})=-\lambda \sin \theta \cdot \ln \left(1-G_{L}\right)
$$

Similarly, in the case of XD by a diffractometer (Fig. 1(b)) where incident and reflecting angles are equal, the equation corresponding to eq. (1) is

$$
\mathrm{d} I_{D} \propto \exp (-2 \mu x / \sin \theta) \cdot \mathrm{d} x
$$

where $\mu$ is the linear absorption coefficient of diffracting X-ray and $\theta$ is the reflection angle. Since $2 d_{h k l} \sin \theta=\lambda_{X}$ which is a well-known relation among the wavelength of the X-ray, $\lambda_{X}$, the lattice spacing with index $(h k l), d_{h k l}$, and the diffraction angle, $\theta$,

$$
L(\mathrm{XD})=-\left(\lambda_{X} / 4 d_{h k l} \cdot \mu\right) \cdot \ln \left(1-G_{L}\right) .
$$

In EPMA, the signal intensity of the characteristic X-ray, corresponding to eqs. (2) and (4), is proportional to $\phi(\rho x) \cdot \exp (-\chi \rho x) \cdot d(\rho x)$ where $\phi$ is the ionization distribution function, 
$\rho$ is the density of the sample, $\chi=(\mu / \rho) \sin \theta$, $\mu$ is the linear absorption coefficient of the analysing X-ray and $\theta$ is the take-off angle. Consequently, the analysing depth for EPMA, $L$ (EPMA), can be obtained from the following relation:

$$
\frac{\int_{0}^{\rho \cdot L(\text { EPMA })} \phi(\rho x) \cdot \exp (-\chi \rho x) \cdot \mathrm{d}(\rho x)}{\int_{0}^{\infty} \phi(\rho x) \cdot \exp (-\chi \rho x) \cdot \mathrm{d}(\rho x)}=G_{L} .
$$

When $\phi(\rho x)$ is known experimentally or theoretically, it is possible to estimate $L$ (EPMA) graphically or analytically. The ionization distribution functions for $K \alpha$ radiation in copper at various accelerating voltages have been reported by Shimizu et al. ${ }^{(13)}$ According to eq. (5), $L$ (EPMA) at the corresponding accelerating voltage is estimated graphically. $\chi=10.8 \mathrm{~m}^{2} / \mathrm{kg}(\operatorname{cosec}=2)$ and $\rho=8.47 \mathrm{Mg} / \mathrm{m}^{3}$ were used for the calculation of $\exp (-\chi \rho x)$. The results are plotted in Fig. 2. It is found that $\alpha=9.068 \times 10^{-4}, \beta=2.002$ and therefore it can be said that $L$ (EPMA) for the $\mathrm{CuK \alpha}$

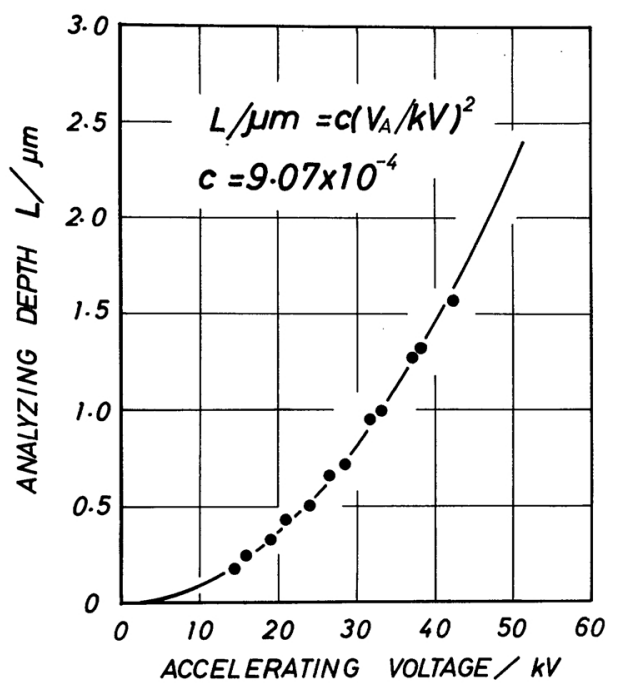

Fig. 2 Relationship between the analysing depth and the accelerating voltage in EPMA. in copper is proportional to the square of the accelerating voltage, $V_{A}$, that is;

$$
L(\text { EPMA }) / \mu m=9.07 \times 10^{-4}\left(V_{A} / \mathrm{kV}\right)^{2} .
$$

It would be reasonable to expect that a similar relation also holds for the $\mathrm{ZnK \alpha}$ in zinc since the atomic number of zinc is very close to copper. The above expression is, therefore, adopted for $L$ (EPMA) in brass. It is also assumed for the $L \alpha$ lines.

The analysing depths thus obtained are shown in Table 1. It implies that the combination of these analysing methods with different analysing depth will give information for a range from $2.4 \mathrm{~nm}$ to $15 \mu \mathrm{m}$.

An analytical composition $\bar{C}(L)$ by an analytical method with an analysing depth $L$ is not equal to the true composition $C(L)$ at the depth $L$, but it should be related to $\bar{C}(L)$ as follows:

$$
\bar{C}(L)=\int_{0}^{\infty} C(x) \cdot \mathrm{d} I(x, L) / \int_{0}^{\infty} \mathrm{d} I(x, L),
$$

where $\mathrm{d} I(x, L)$ is the signal intensity from a thin slab with thickness $\mathrm{d} x$ and a unit concentration of a given substance at depth $x$. Taking $\mathrm{d} I(x, L)=Y(x, L) \cdot \mathrm{d} x$, eq. (7) is rewritten as

$$
\bar{C}(L)=\int_{0}^{\infty} C(x) \cdot Y(x, L) \mathrm{d} x / \int_{0}^{\infty} Y(x, L) \mathrm{d} x .
$$

In the case of XAES and XD, the representation of $Y(x, L)$ is

$$
Y=\exp \left\{x \ln \left(1-G_{L}\right) / L\right\} .
$$

For EPMA,

$$
Y=\phi(\rho x, L) \exp (-\chi \rho x) .
$$

It may be possible to approximate the true composition at each analysing depth $L$ from the value observed by the analysing method with the analysing depth $L$, but a better approximation would be attained by considering ionization function and absorption. $C(L)$ can

Table 1 Analysing depth, $L$, in $\alpha$-brass.

\begin{tabular}{ccccccccc}
\hline \multirow{2}{*}{ Method } & XAES & \multicolumn{4}{c}{ EPMA } & \multicolumn{2}{c}{ XD } \\
\cline { 2 - 9 } & $L_{3} M_{4,5} M_{4,5}$ & $5 \mathrm{kV}$ & $10 \mathrm{kV}$ & $15 \mathrm{kV}$ & $25 \mathrm{kV}$ & $50 \mathrm{kV}$ & $\mathrm{Cr} K \alpha$ & $\mathrm{Cu} K \alpha$ \\
\hline$L / \mu \mathrm{m}$ & $2.39 \times 10^{-3}$ & $2.27 \times 10^{-2}$ & $9.11 \times 10^{-2}$ & 0.205 & 0.571 & 2.27 & 4.74 & 14.9 \\
\hline
\end{tabular}


be obtained by the inverse transformation of eq. (8). It might be approximately attained by several methods. In this report, the problem is reduced to solve eight simultaneous equations in eight unknowns of the actual compositions since eight observed values are obtained by measurements. Taking the composition at each analysing depth as each unknown, it is assumed that the composition varies linearly between the neighboring analysing depths. Under this assumption, two possible models are proposed. One is that the composition at the top surface is equal to that at the smallest analysing depth (MODEL 1 in Fig. 3), and the other is that the composition at the deepest analysing depth is equal to the bulk composition (MODEL 2 in Fig. 3). In both models, $L_{9}$ is equal to $10 \mathrm{~mm}$ which is taken as a representative depth with bulk composition.

Under these models, eq. (8) can be expressed approximately as follows, since $Y\left(x, L_{j}\right)$ is
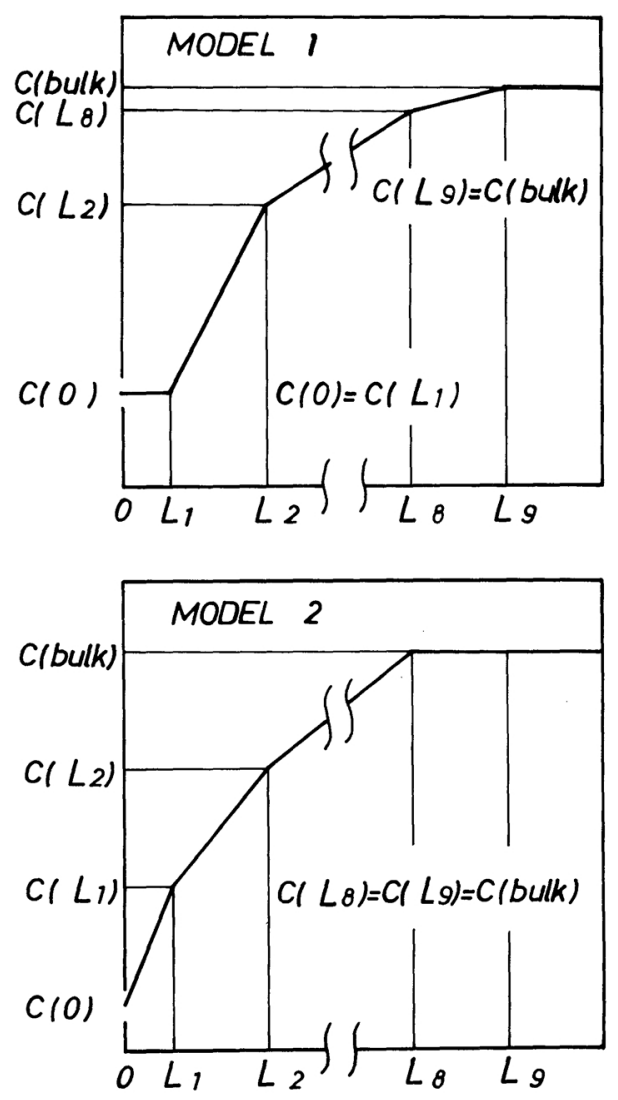

Fig. 3 Composition distribution models. negligibly small at such a large $x$ as $10 \mathrm{~mm}$ :

$$
\begin{aligned}
\bar{C}\left(L_{j}\right)= & {\left[\sum _ { i = 0 } ^ { 9 } \int _ { L _ { i } } ^ { L _ { i + 1 } } \left\{\left(C\left(L_{i}\right)+\left(\frac{C\left(L_{i+1}\right)-C\left(L_{i}\right)}{L_{i+1}-L_{i}}\right)\right.\right.\right.} \\
& \left.\left.\cdot\left(x-L_{i}\right)\right\} \cdot Y\left(x, L_{j}\right) \mathrm{d} x\right] / S,
\end{aligned}
$$

where $L_{0}=0, j$ denotes an analysing method with an analysing depth $L_{j}$ and

$$
S=\sum_{i=0}^{9} \int_{L_{i}}^{L_{i+1}} Y\left(x, L_{j}\right) \cdot \mathrm{d} x .
$$

Model 1 assumes $C(0)=C\left(L_{1}\right)$ and $C\left(L_{i, \max }\right) \neq$ $C\left(\right.$ bulk ) and Model 2, $C(0) \neq C\left(L_{1}\right)$ and $C\left(L_{i, \max }\right)=C($ bulk $)$. In this case, $i, \max =8$. Consequently, eq. (11) represents a set of eight simultaneous equations in eight unknowns $\left(C\left(L_{i}\right), i=1, \cdots, 8\right.$ for Model 1 and $i=0, \cdots$, 7 for Model 2) and the coefficients in those equations can be obtained when $Y\left(x, L_{j}\right)$ is defined.

In the case of EPMA data, eq. (11) involves $\phi(\rho x) \cdot \exp (-\chi \rho x)$ as shown in eq. (10). It would be more convenient to deal with a simple analytical function instead of the reported graph of $\phi(\rho x) . Y(x, L)$ for EPMA is, therefore, approximated by the simplest analytical function which has the same characteristic features as $\phi(\rho x) \cdot \exp (-\chi \rho x)$ graphically obtained from the ionization function reported by Shimizu et al. ${ }^{(13)} \phi(\rho x) \cdot \exp (-\chi \rho x)$ graphically obtained has several characteristic features. One of them is that

$$
\begin{aligned}
& \int_{0}^{x_{a}} \phi(\rho x) \cdot \exp (-\chi \rho x) d(\rho x) \\
& \simeq \frac{7}{9} \int_{0}^{\infty} \phi(\rho x) \cdot \exp (-\chi \rho x) \mathrm{d}(\rho x)
\end{aligned}
$$

at various accelerating voltages where $\phi(\rho x)$. $\exp (-\chi \rho x)$ has a half maximum value at $x=x_{a}$. This is the reason why $7 / 9$ is chosen for $G_{L}$. Including this nature, the main charactereistic features which should be carried by the function $Y(x, L)$ for EPMA are abstracted from the term $\phi(\rho x) \cdot \exp (-\chi \rho x)$ graphically obtained. They are summarized as follows:

(i) $\int_{0}^{Z_{a}} Y \mathrm{~d} Z=\frac{7}{9} \int_{0}^{\infty} Y \mathrm{~d} Z$

where

$$
Z=\rho x \text { and } Z_{a}=\rho L,
$$


(ii) $Y$ has a maximum value at $Z=B$ which is proportional to $Z_{a}$ and $0<B<Z_{a}$,

(iii) $Y(B)=2 Y\left(Z_{a}\right)$, and

(iv) $Y$ decreases exponentially to zero at large $Z$.

$Y$ for EPMA approximated by the simplest analytical function which fulfills the above requirements is as follows:

$$
\begin{aligned}
Y(\text { EPMA })= & \left(Z-Z_{a}\right)\left\{\left(Z-Z_{a}\right)^{2}-3\left(Z_{a}-B\right)^{2}\right\} \\
& +2\left(Z_{a}-B\right)^{3} \text { for } 0 \leq Z \leq Z_{a} \\
= & 2\left(Z_{a}-B\right)^{3} \exp \left\{-3\left(Z-Z_{a}\right) / 2\left(Z_{a}\right.\right. \\
& -B)\} \text { for } Z \geq Z_{a},
\end{aligned}
$$

where $B$ is determined from $9 \int_{0}^{Z_{a}} Y \mathrm{~d} Z=7 \int_{0}^{\infty} Y \mathrm{~d} Z$ and is equal to $0.3677 Z_{a}$. This value of $B$ was nearly equal to the real value estimated from the peak position of $Y$ from $\phi(\rho x)$ by Shimizu et al. ${ }^{(13)}$ Now, the coefficients of eight simultaneous equations in eight unknowns can be easily calculated.

\section{Results and Discussion}

By the XAES method, the existence of oxide film 2-3 nm thick was recognized and was considered for the determination of the underlying alloy composition. In EPMA and XD, the existence of the oxide film was disregarded since its thickness is negligibly small in comparison with the analysing depth of these methods. The weight percent of zinc in samples $A$ and $B$ at each depth was obtained by solving the eight simultaneous equations. Results concerning samples $\mathrm{A}$ and $\mathrm{B}$ are plotted against depth in Fig. 4 and Fig. 5, respectively. It can be seen that the solution under each model mentioned in the preceding section gives a zigzag pattern and those models are complementary. The difference between the obtained values under two models is, at most, about 4 and $6 \%$ at each analysing depth for samples $\mathrm{A}$ and $\mathrm{B}$, respectively. The difference always appears alternatively in both samples. This fact suggests that this alternative deviation may arise from the characteristic of the two opposite extreme models. An intermediate model between these extremely opposite models would be favourable. Therefore, in Fig. 6, the means of the results obtained from both models are

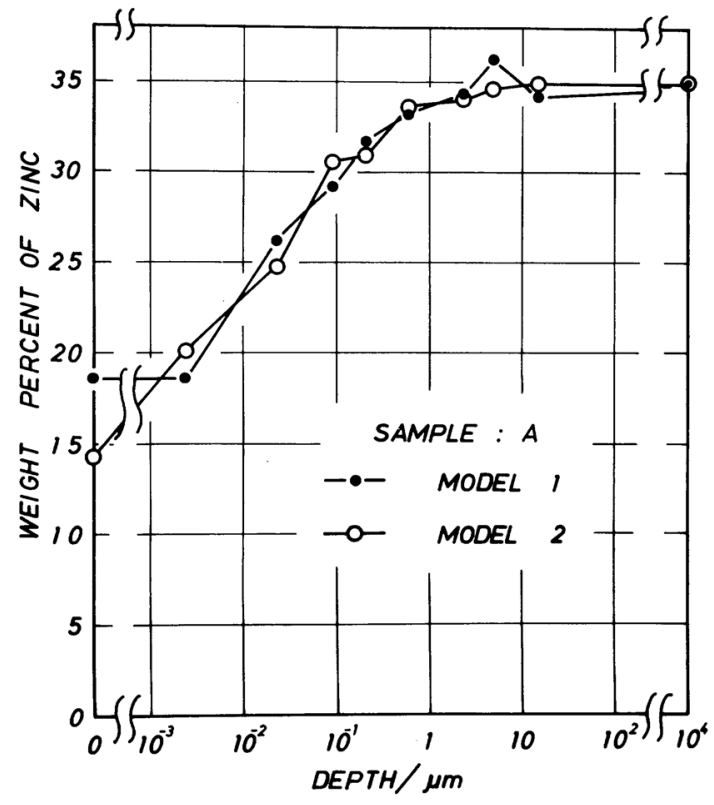

Fig. 4 Comparison of depth profiles of $\mathrm{Zn}$ in sample A corrected under two extreme models.

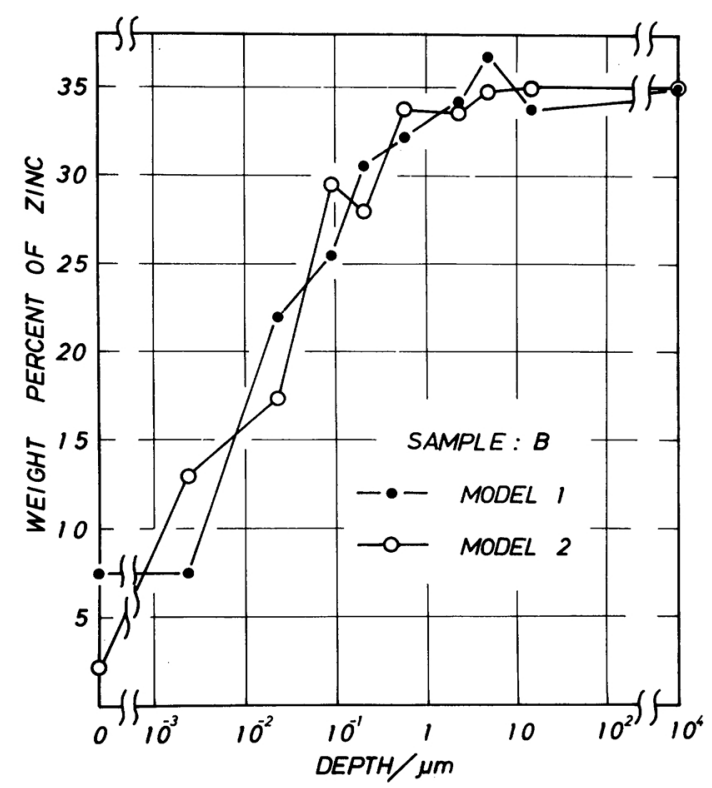

Fig. 5 Comparison of depth profiles of $\mathrm{Zn}$ in sample B corrected under two extreme models.

plotted against the depth as "corrected". For comparison, the observed values are also plotted in Fig. 6 versus analysing depth as "measured". By taking the average value between two models, the corrected results fall 


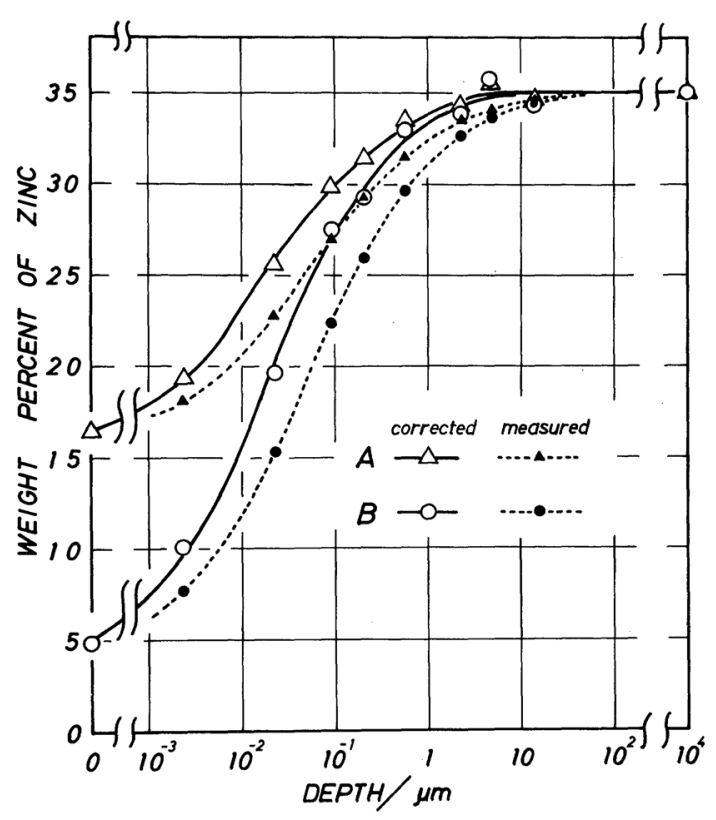

Fig. 6 Comparison of the measured and corrected depth profiles of $\mathrm{Zn}$ in $\alpha$-brass.

on relatively smooth curves. In both samples, severe dezincification is observed at depth less than $1 \mu \mathrm{m}$ and is very large in sample B. This depencence is also shown in the plot of "measured" values. The correction is always possitive for the zinc content. This can be understood because the zinc content is lower at the smaller depth. The correction becomes largest in the depth range from $10^{-2}$ to $10^{-1} \mu \mathrm{m}$. The degree of correction may depend on the compositiondepth profile since the correction is made with respect to the nonuniformity of element distribution.

The value of $G_{L}$ in this report has happened to be $7 / 9$ as a matter of convenience. Another choice of $G_{L}$ would be possible if more favorable. The different choice of $G_{L}$ naturally leads to change in the value of the analysing depth $L$. It would, therefore, bring about a lateral shift of the plot of the concentration measured in Fig. 6 corresponding to the change in $L$. However, the corrected values are not influenced much because $Y(x, L)$ itself does not depend on $G_{L}$ but, essentially rather on the analysing method and condition directly though formally it contains $L$ as a parameter. In the real concentration-depth profile representation,
$L$ is used only as for a parameter describing the profile. It is also possible to use a depth parameter other than $L$. Since the depth profile is approximated with linear functions as shown in Fig. 3, it would be favorable to take a depth parameter distributed closely at the parts of small curvature of the concentration-depth profile. However, the analysing depth $L$ could be the most practical and proper choice as a depth parameter under the conditions that the number of the experimental data is limited and the first approximation of the depth profile is expressed by the plot of these data directly against $L$.

In the estimation of analysing depth for the EPMA measurement using the $L \alpha$ lines, the relationship (6) was adopted which is for the $\mathrm{Cu} K \alpha$ lines in copper. The absorption for the $L \alpha$ lines are larger than that for the $K \alpha$ lines. Therefore, the true analysing depth for the $L \alpha$ lines would be less than the values in Table 1. However, it should not be so much less since an analysing depth which was one order of magnitude smaller would give irregular discontinuity on the plotted curves of measured values against the analysing depth. Such discontinuity is highly improbable in these samples considering their production process.

In this analysis, the differences in absorption of X-rays from place to place and between different characteristic X-rays for EPMA are ignored since it is not significant in brass because of neighboring atomic numbers of the constituent elements. Generalization of this method would require that the difference in the absorption should be taken into account. One of the approaches to the solution of this problem is to make a plot of the measured values against the analysing depth as a starting point of the gradual approximation; to estimate the distribution of the absorption coefficient as the first approximation and so on. It will be a much complicated procedure but may be possible by use of recent computer techniques.

Copper and zinc, the constituent elements of brass, are special since they have smaller chemical shifts of X-ray photoelectron (XPS) and larger chemical shifts of AES spectra. Instead of XAES, usual XPS could be used in cases where the chemical shifts of XPS are 
large enough to be distinguished which would be rather familiar in XPS of ordinary solid solution alloy systems.

\section{Conclusion}

(1) Non-destructive in-depth profile ranging from nanometer to tens of micrometer is possible for single-phase brass by a combined use of several analysing methods with different analysing depths such as X-ray induced Auger electron spectroscopy, electron microprobe analysis and X-ray diffraction.

(2) The analysing depth of EPMA by the $K \alpha$ lines in brass is proportional to the square of the accelerating voltage.

(3) The observed value by an analysing method with a certain analysing depth does not represent the real composition at the depth but should be corrected considering the composition distribution. The most probable true composition profile can be estimated by taking the average of the values obtained under the two extreme models of compoistion profile; one assumes $C(0)=C\left(L_{1}\right)$, and $C\left(L_{i, \text { max }}\right) \neq$ $C$ (bulk) and the other, $C(0) \neq C\left(L_{1}\right)$ and $C\left(L_{i, \max }\right)=C($ bulk $)$, and in both models,

$$
C(x)=C\left(L_{i}\right)+\frac{C\left(L_{i+1}\right)-C\left(L_{i}\right)}{L_{i+1}-L_{i}} \cdot\left(x-L_{i}\right)
$$

(4) Samples showed large dezincification at depth less than $1 \mu \mathrm{m}$. The largest correction corresponding to those composition profiles appeared in the depth range between $10^{-2}$ and $10^{-1} \mu \mathrm{m}$.

\section{Acknowledgment}

The author wishes to thank Mr. S. Oki for $\mathrm{X}$-ray diffraction measurement.

\section{REFERENCES}

(1) H. Shimizu, M. Ono and K. Nakayama: Surface Sci., 36 (1973), 817.

(2) L. A. Harris: Surface Sci., 15 (1969), 77.

(3) C. S. Fadley, R. J. Baird, W. Siekhaus, T. Novakov and S. Å. L. Bergstrom: J. Electron Spectrosc., 4 (1974), 93.

(4) K. Asami, K. Hashimoto and S. Shimodaira: Trans. JIM, 19 (1978), 598.

(5) S. Storp and R. Holm: Proc. 7th Intern. Vac. Congr. \& 3rd Intern. Conf. Solid surfaces (Vienna 1977), p. 2255.

(6) C. R. Houska: J. Appl. Phys., 41 (1970), 69.

(7) D. R. Tenney, J. A. Carpenter and C. R. Houska: J. Appl. Phys., 41 (1970), 4485.

(8) J. A. Carpenter, D. R. Tenney and C. R. Houska: J. Appl. Phys., 42 (1971), 4305.

(9) S. Sato: Bull. Japan Inst. Met., 15 (1976), 53.

(10) K. Asami and K. Hashimoto: Trans. JIM, 20 (1979), 119.

(11) L. H. Beck and C. S. Smith: J. Metals, 5 (1952), 1079.

(12) K. Asami, K. Hashimoto and S. Shimodaira: J. Japan Inst. Metals, 40 (1976) 438.

(13) R. Shimizu, K. Murata and G. Shinoda: Figure 8 in Bull. Res. Inst. Mineral Dress. and Metallur. Tohoku Univ., 21 (1965), 171, by K. Sambongi and Y. Omori. 